\title{
Constructing a Pareto Front Approximation for Decision Making
}

\author{
Markus Hartikainen - Kaisa Miettinen . \\ Margaret M. Wiecek
}

Received: date / Accepted: date

\begin{abstract}
An approach to constructing a Pareto front approximation to computationally expensive multiobjective optimization problems is developed. The approximation is constructed as a sub-complex of a Delaunay triangulation of a finite set of Pareto optimal outcomes to the problem. The approach is based on the concept of inherent nondominance. Rules for checking the inherent nondominance of complexes are developed and applying the rules is demonstrated with examples. The quality of the approximation is quantified with error estimates. Due to its properties, the Pareto front approximation works as a surrogate to the original problem for decision making with interactive methods.
\end{abstract}

Keywords Multiobjective Optimization · Multiple Criteria Decision Making · Pareto Optimality · Interactive Decision Making · Interpolation · Delaunay Triangulation

\section{Introduction}

In practical decision problems, there are often multiple conflicting objectives that need to be optimized at the same time (Keeney and Raiffa, 1993). These conflicting objectives are often handled with ad hoc aggregation or by converting all but one objective into constraints. Multiobjective optimization is a systematic approach to optimizing multiple conflicting objectives (see e.g., (Miettinen, 1999)).

Different multiobjective optimization methods can be classified into four classes with respect to the role of a decision maker (an expert in the application area who is at liberty to make decisions concerning the problem) in the optimization process

This research was partly supported by the Academy of Finland grant number 128495.

Markus Hartikainen · Kaisa Miettinen · Margaret M. Wiecek*

Department of Mathematical Information Technology,

University of Jyväskylä, Finland

${ }^{*}$ On sabbatical leave from Clemson University, Clemson, SC, USA

E-mail: markus.e.hartikainen@jyu.fi·kaisa.miettinen@jyu.fi ·wmalgor@clemson.edu 
(Miettinen, 1999; Sawaragi et al., 1985). In no-preference methods, no preference information is used. In a priori methods, the problem is optimized with respect to a given preference information and then the decision maker is provided with a single solution. In a posteriori methods, the decision maker is provided with a set of solutions from which he/she can choose a preferred one. In interactive methods, an iterative procedure is used to explore different solutions to the problem and the decision maker is allowed guide the exploration. All these methods find their use in different situations, but we have concentrated on interactive methods because they allow the decision maker to learn about the problem while solving it (Miettinen et al., 2008). In all methods, an important concept is Pareto optimality. A solution to a multiobjective optimization problem is Pareto optimal, if none of the objectives can be improved without impairing some other(s). An outcome is a vector containing the values of objectives as its components, and an outcome is Pareto optimal if it is given by a Pareto optimal solution. The preferences are in interactive methods typically (see (Luque et al., 2011)) given in the form of desired values, changes or proportions of the values of objectives in Pareto optimal outcomes (see e.g., the NIMBUS method (Miettinen and Mäkelä, 1995, 2000, 2006) and the Tchebycheff method (Steuer and Choo, 1983; Steuer, 1986, 1989) to name some).

In addition to having conflicting objectives, many practical decision problems are also computationally expensive (see e.g., (Hasenjäger and Sendhoff, 2005; Laukkanen et al., 2010)). The computational cost results from the lack of closed-form description of the objective functions and the need to employ simulation, which may take even hours (see e.g., (Hasenjäger and Sendhoff, 2005)). Computationally expensive problems are naturally very hard to solve and even interactive methods - that are often praised for their computational efficiency when compared to a posteriori methods (Miettinen, 1999) - may be inefficient. This is because the time grows between the moments when the decision maker expresses his/her preferences and when he/she can see the effects of those preferences in generated outcomes. The time grows because in most interactive methods the multiobjective optimization problem is in each iteration solved with respect to the updated preferences of the decision maker. This naturally slows down the solution process and makes it harder for the decision maker to learn about the problem and may even make him/her reluctant to explore different solutions to the problem.

We have developed an approach to help interactive methods deal with computationally expensive problems. The approach is based on replacing the original problem with a surrogate problem which approximates the set of Pareto optimal outcomes (often also called the Pareto front). Following this idea, we proposed in (Hartikainen et al., 2011) that one should construct (as defined therein) an inherently nondominated Pareto front approximation based on a small set of known Pareto optimal outcomes and use this approximation with the interactive method instead of the original problem. The reasoning behind an inherently nondominated Pareto front approximation is that we construct a set in the space of outcomes that contains the known Pareto optimal outcomes as its subset. In this way, we can examine also other possible Pareto optimal outcomes that are not contained in the set of known Pareto optimal outcomes. In (Hartikainen et al., 2011), we argued that an inherently nondominated Pareto front approximation does not include objective function values that would mislead the de- 
cision maker. To be more specific, when approximating the Pareto front of a nonconvex multiobjective optimization problem, convex approximations (e.g., convex hulls or ellipses) may include vectors that are strictly better in all components than a known Pareto optimal outcome that was used to construct this approximation. These vectors are infeasible for the problem by definition of Pareto optimality. One of the benefits of an inherently nondominated approximation is that it avoids this behavior.

An inherently nondominated Pareto front approximation gives rise to a multiobjective optimization problem that can in a meaningful way be seen as a surrogate for the original problem. The Pareto front of the surrogate problem equals the inherently nondominated approximation and solving this problem interactively can be seen as exploring the approximation with interactive methods. This surrogate problem has a closed form formulation and, thus, it is computationally much less expensive than the original problem. The construction of the approximation unavoidably requires computation, but it can be done before involving the decision maker. After the approximation has been constructed, the decision maker can use the interactive method of his/her choice with the surrogate problem without computational delay. When a preferred outcome for the surrogate problem is found, it can then be projected onto the actual Pareto front of the original problem with e.g., an achievement scalarizing function (Wierzbicki, 1986).

Approaches similar to ours, where a Pareto front approximation is used in decision making, are given in (Eskelinen et al., 2010; Lotov et al., 2004; Monz, 2006). Eskelinen et al. (2010) and Monz (2006) use custom-made iterative procedures for finding a preferred element on the approximation, while we develop an approximation that can be used with almost any interactive method. The benefit of being able to use various interactive methods is that we can support diverse decision makers who may prefer different methods. In addition, approaches in (Eskelinen et al., 2010; Monz, 2006) are only applicable to convex multiobjective optimization problems, while ours can handle both convex and nonconvex problems. The approach in (Lotov et al., 2004) differs from ours, because it relies on a visualization technique called Interactive Decision Maps to find a preferred element on the approximation.

Methods for constructing Pareto front approximations (published before the year 2003) are surveyed in (Ruzika and Wiecek, 2005) and more recent ones include (Bezerkin et al., 2006; Efremov and Kamenev, 2009; Goel et al., 2007; Martin et al., 2005). Some of these methods are not applicable for our needs, because they cannot create a Pareto front approximation for nonconvex multiobjective optimization problems. Other methods cannot operate based on a given set of Pareto optimal outcomes, but assume that some specific a posteriori method has been used to generate the outcomes. The value added by being able to use a given set of Pareto optimal outcomes is that one can use Pareto optimal outcomes generated with any a posteriori method (see e.g., (Miettinen, 1999)) including evolutionary multiobjective optimization methods (see e.g., (Deb, 2001; Coello Coello et al., 2007)). Furthermore, none of the methods in the literature guarantee producing an inherently nondominated Pareto front approximation.

In this paper, we lay theoretical foundations for constructing an inherently nondominated Pareto front approximation for (convex or nonconvex) multiobjective optimization problems with continuous objectives. The proposed approximation im- 
proves on the weaknesses of existing methods discussed above. Technically, our approximation is based on intelligent interpolation between a set of known Pareto optimal outcomes. The interpolants are taken from the polytopes of a Delaunay triangulation (see e.g., (Fortune, 1997)) of the set of known Pareto optimal outcomes. The idea for our approximation comes from computational geometry (for a general overview see e.g., (Goodman and O'Rourke, 1997)), where different subcomplexes of Delaunay triangulations have been extensively used in shape reconstruction (Edelsbrunner, 1998). We construct a subcomplex that satisfies some properties that are based on inherent nondominance so that the final approximation is inherently nondominated. The Delaunay triangulation is of combinatorial nature. Here we do not deal with the implementation of our approximation but concentrate on the theoretical aspects.

The rest of this paper is structured as follows. Section 2 concentrates on the notations and basic definitions used in this paper. The most relevant concepts from computational geometry are presented in Section 3 and combined with the inherent nondominance property in Section 4 . Section 5 proposes an approach to constructing an inherently nondominated Pareto front approximation as a sub-complex of a Delaunay triangulation while Section 6 demonstrates the proposed approximation with two examples. Properties of the approximation for two special types of multiobjective optimization problems are examined in Section 7 and error estimates for the approximation are derived in Section 8. Finally, Section 9 concludes with some remarks on decision making with the constructed Pareto front approximation.

\section{Notation and Definitions}

We consider multiobjective optimization problems

$$
\min _{\text {s.t. } x \in S}\left(f_{1}(x), \ldots, f_{k}(x)\right),
$$

where $f_{i}: S \rightarrow \mathbb{R}$ is for all $i \in\{1, \ldots, k\}$ a real-valued function and $S \subset \mathbb{R}^{n}$. We define a vector-valued function $f: S \rightarrow \mathbb{R}^{k}, f(x)=\left(f_{1}(x), \ldots, f_{k}(x)\right)^{T}$ for all $x \in S$. Because we aim to interpolate between the known Pareto optimal outcomes, we assume that all objectives are measured in continuous scales. This is the case for example when the set $S$ is connected and the functions $f_{i}$ are continuous.

The set $S$ is called the feasible decision set of the multiobjective optimization problem and every vector $x \in S$ is called a (feasible) decision. The set $f(S)$ is the feasible outcome set of the multiobjective optimization problem and every vector $z \in f(S)$ is called a (feasible) outcome.

For a set $K \subset \mathbb{R}^{k}$ the boundary of $K$ is denoted by bnd $(K)$ and the interior is denoted by $\operatorname{int}(K)$. The closure of the set $K$ is denoted by $\operatorname{cl}(K)$. The convex hull of $K$ is denoted by $\operatorname{conv}(K)$. For two sets $K^{1}, K^{2} \subset \mathbb{R}^{k}$ the notation $K^{1} \subset K^{2}$ means that if a vector $s \in K^{1}$ then $s \in K^{2}$. Notation $K^{1} \subsetneq K^{2}$ means that $K^{1} \subset K^{2}$ but $K^{1} \neq K^{2}$.

For two vectors $z^{1}, z^{2} \in \mathbb{R}^{k}$, notation $z^{1} \leq z^{2}$ means that $z_{i}^{1} \leq z_{i}^{2}$ for all $i=1, \ldots, k$ and $z^{1} \neq z^{2}$. If $z^{1} \leq z^{2}$ then $z^{1}$ is said to dominate $z^{2}$ or $z^{2}$ is said to be dominated by $z^{1}$. A vector $b \in B$ is Pareto optimal in a set $B \subset \mathbb{R}^{k}$ if there does not exist a vector $b^{\prime} \in B$ that dominates $b$. An outcome $z \in f(S)$ is Pareto optimal for multiobjective 
optimization problem (1) if the vector $z$ is Pareto optimal in the set $f(S)$. Finally, the Pareto front of the set $B$, denoted by $\operatorname{PF}(B)$, is the set of Pareto optimal vectors in the set $B$ and the Pareto front of (1), denoted by PF, is the set of Pareto optimal outcomes of the multiobjective optimization problem. A feasible decision $x \in S$ is a Pareto optimal solution to the multiobjective optimization problem if $f(x)$ is a Pareto optimal outcome for the multiobjective optimization problem.

In (Hartikainen et al., 2011), some properties and definitions concerning Pareto front approximations are given. They are summarized below.

Definition 1 A set $A \subset \mathbb{R}^{k}$ is inherently nondominated if there do not exist vectors $a, b \in A$ so that $a \leq b$.

Definition 2 Let $P \subset \mathbb{R}^{k}$ be a finite set of Pareto optimal outcomes. A set $A \subset \mathbb{R}^{k}$ is an inherently nondominated Pareto front approximation (based on the set $P$ ) if the set $A$ is inherently nondominated and $P \subset A$.

Definition 3 Let $B \subset \mathbb{R}^{k}$ be a set and let the set $P \subset \mathbb{R}^{k}$ be as above. A set $A \subset B$ is a $B$-maximal inherently nondominated Pareto front approximation (based on the set $P$ ) if the set $A$ is an inherently nondominated Pareto front approximation based on $P$ and for all vectors $b \in B \backslash A$ the set $A \cup\{b\}$ is not inherently nondominated.

As shown in (Hartikainen et al., 2011), an inherently nondominated Pareto front approximation based on $P$ is the Pareto front of a multiobjective optimization problem that has outcomes $P$ on its Pareto front. In this way, an inherently Pareto front approximation can be seen as a surrogate for the actual Pareto front.

The starting point of this study is as follows. We assume that we are given a computationally expensive multiobjective optimization problem. This problem is referred to as the initial multiobjective optimization problem. We also assume that we are given a finite set of $m$ Pareto optimal outcomes $P=\left\{p^{1}, \ldots, p^{m}\right\}$. This set is referred to as the initial set of Pareto optimal outcomes or the known Pareto optimal outcomes. This set may have been generated with any appropriate method in the literature e.g., an evolutionary multiobjective optimization method (Deb, 2001) or any of the $0^{\text {th }}$-order approximation methods mentioned in (Ruzika and Wiecek, 2005). In this paper, we use the initial set of Pareto optimal outcomes for constructing an inherently nondominated Pareto front approximation that intelligently interpolates between the known Pareto optimal outcomes.

\section{Background on Polytopes, Complexes and (Delaunay) Triangulations}

The Pareto front approximation constructed in this paper is a complex i.e., a collection of polytopes with certain properties. In the first part of this section, we review all the properties of polytopes and complexes that are needed in this paper. For the proofs and further properties we refer to (Grünbaum, 1967) and references therein. We mostly follow the notation and definitions established in (Grünbaum, 1967). The only difference is that here the prefix " $a$-" before the words "polytope", "face" or "complex" refers to the number of vertices minus one and not to the dimension. This 
is because in our setting it is easier to count the number of vertices of the polytope than to compute the dimension of the polytope.

The rest of this section deals with triangulations of a finite vector set. A triangulation is a complex with certain additional properties. Especially, we discuss the Delaunay triangulation, which is a well-known triangulation (see e.g., (Edelsbrunner and Shah, 1994; Fortune, 1997)). Some reasons for the fame of the Delaunay triangulation are that it is useful in shape reconstruction, as argued in (Boissonnant, 1984 ), and it can be computed in $\mathbb{R}^{k}$ by computing the convex hull of a set in $\mathbb{R}^{k+1}$, as shown in (Edelsbrunner, 1987). In our approximation, the complex that approximates the Pareto front is a sub-complex of a Delaunay triangulation of the initial set of Pareto optimal outcomes $P$. Previously Delaunay triangulations have been used mostly in finite element methods (see e.g., (George and Borouchaki, 1998; Shenton and Cendes, 1985)) and for reconstructing different solids from a set of vectors (see e.g., (Edelsbrunner, 1998)). In (Schandl et al., 2002), the Delaunay triangulation is used for initializing an algorithm for Pareto front approximation. Their aim is to construct a piecewise linear approximation by means of block norms whose level sets provide a polyhedral structure of the approximation. However, the resulting approximation has features different from ours and does not allow the decision maker to use interactive multiobjective optimization methods.

An alternative comprehensive treatment of the topics contained in this section can be found in (Fukuda, 2004). That article also contains references to more detailed treatments of these and further topics.

Definition 4 Let $a \in \mathbb{N}$. The set

$$
\mathscr{P}\left(z^{1}, \ldots, z^{a+1}\right)=\operatorname{conv}\left(\left\{z^{1}, \ldots, z^{a+1}\right\}\right)
$$

with vectors $z^{1}, \ldots, z^{a+1} \in \mathbb{R}^{k}$ is called a (convex $a$-)polytope. The empty set is a $(-1)$-polytope. The polytope $\mathscr{P}\left(z^{1}, \ldots, z^{a+1}\right)$ is said to be determined by vectors $z^{1}, \ldots, z^{a+1}$

The following propositions and definitions summarize the properties of polytopes. The proofs can be found in (Grünbaum, 1967).

Proposition 1 Let $a, b \in \mathbb{N}$ and consider vectors $z^{1}, \ldots, z^{a+1}, \hat{z}^{1}, \ldots, \hat{z}^{b+1} \subset \mathbb{R}^{k}$. If $\left\{z^{1}, \ldots, z^{a+1}\right\} \subset\left\{\hat{z}^{1}, \ldots, \hat{z}^{b+1}\right\}$, then $\mathscr{P}\left(z^{1}, \ldots, z^{a+1}\right) \subset \mathscr{P}\left(\hat{z}^{1}, \ldots, \hat{z}^{b+1}\right)$.

Definition 5 A vertex of a polytope is a vector $x \in K$ so that if $y, z \in K, 0<\lambda<1$ and $x=\lambda y+(1-\lambda) z$, then $x=y=z$. The set of all vertices is denoted by vert $(K)$.

Definition 6 Let $u \in R^{k}$ be a vector, $\alpha \in \mathbb{R}$ be a scalar and $K \subset \mathbb{R}^{k}$ be a polytope. The hyperplane

$$
H=\left\{x \in \mathbb{R}^{k}: x^{T} u=\alpha\right\}
$$

is a supporting hyperplane of the polytope $K$ if $K \cap H \neq \emptyset$ and either $K \subset\left\{x \in \mathbb{R}^{k}\right.$ : $\left.x^{T} u \leq \alpha\right\}$ or $K \subset\left\{x \in \mathbb{R}^{k}: x^{T} u \geq \alpha\right\}$. 
Definition 7 Let $K \subset \mathbb{R}^{k}$ be a polytope. A set $F \subset \mathbb{R}^{k}$ is called a face of the polytope $K$ if $F=\emptyset, F=K$ or if there exists a supporting hyperplane $H$ of the polytope $K$ so that $F=K \cap H$. The sets $\emptyset$ and $K$ are called improper faces of the polytope $K$ and the other faces are called proper faces of $K$. A face $F$ that is also an $a$-polytope is called an $a$-face.

Example 1 If $K=\mathscr{P}\left(z^{1}, z^{2}\right) \subset \mathbb{R}^{3}$ is a 1-polytope with $z^{1} \neq z^{2}$, then the set of vertices is $\operatorname{vert}(K)=\left\{z^{1}, z^{2}\right\}$ and the faces are $\emptyset,\left\{z^{1}\right\},\left\{z^{2}\right\}$ and $\mathscr{P}\left(z^{1}, z^{2}\right)$.

Proposition 2 A face of a polytope is also a polytope.

Proposition 3 If $K \subset \mathbb{R}^{k}$ is a polytope, then the boundary of the polytope is $\operatorname{bnd}(K)=$ $\cup_{F \in \mathscr{F}} F$, where $\mathscr{F}$ is a collection containing all the proper faces of $K$.

Proposition 4 Let $K^{1}, K^{2}, K^{3} \subset \mathbb{R}^{k}$ be polytopes so that the polytope $K^{2}$ is a face of the polytope $K^{1}$ and the polytope $K^{3}$ is a face of the polytope $K^{2}$. Then the polytope $K^{3}$ is a face of the polytope $K^{1}$.

Definition 8 The dimension of a polytope $K \subset \mathbb{R}^{k}$ is the minimal dimension of a linear subspace $L \subset \mathbb{R}^{k}$ so that $K \subset z+L$ with some $z \in \mathbb{R}^{k}$.

Proposition 5 The dimension of an a-polytope $K \subset \mathbb{R}^{k}$ is at most a.

A simplex is a special type of polytope. In a way, a simplex can be seen as the simplest type of all polytopes, as argued in (Grünbaum, 1967). In order to define a simplex, we need to define affine independence of vectors in $\mathbb{R}^{k}$.

Definition 9 Vectors $v^{1}, \ldots, v^{s} \in \mathbb{R}^{k}$ are affinely independent if the vectors $v^{2}-v^{1}, \ldots$, $v^{s}-v^{1}$ are linearly independent.

Definition 10 An $a$-polytope $K=\mathscr{P}\left(z^{1}, \ldots, z^{a+1}\right)$ is called an $a$-simplex (or merely a simplex) if the vectors $z^{1}, \ldots, z^{a+1}$ are affinely independent.

By Proposition 5, the dimension of an $a$-polytope is at most $a$. We can say even more about simplices.

Proposition 6 The dimension of an a-simplex is a.

Another important property of a simplex is that its faces are also simplices and that any proper subset of vertices determines a proper face of the simplex. Notice that the latter is not true all for polytopes, because e.g., the vertices $(0,0),(1,1)$ of a polytope $\mathscr{P}((0,0),(1,0),(0,1),(1,1)) \subset \mathbb{R}^{2}$ do not determine a face of the polytope.

Proposition 7 Let $a, b \in \mathbb{N}$ so that $a \geq b$. All the $b$-faces of an a-simplex are $b$ simplices and any $b+1$ vertices of a simplex determine $a b$-face of the simplex.

A complex is a collection of polytopes with certain properties. The complex is used to describe polyhedral sets beyond polytopes.

Definition 11 A collection $\mathscr{K}$ of polytopes in $\mathbb{R}^{k}$ is called a (polyhedral) complex provided that 
(1) every face of a polytope in $\mathscr{K}$ is also a polytope in $\mathscr{K}$ and

(2) the intersection of any two polytopes in $\mathscr{K}$ is a face of each of them.

A complex $\mathscr{K}$ is called an $a$-complex, if there exists an $a$-polytope $K \in \mathscr{K}$ and there is no $b$-polytope $K^{\prime} \in \mathscr{K}$ with $b>a$.

The body of a complex contains all the vectors in all the polytopes in the complex. The body is sometimes also called the underlying space (see e.g., (Vegter, 1997)).

Definition 12 The body of a complex $\mathscr{K}$ is $\operatorname{body}(\mathscr{K})=\cup_{K \in \mathscr{K}} K$.

Now we are ready to define a triangulation of a finite set $P \subset \mathbb{R}^{k}$. Informally, the triangulation of a set can be seen as a subdivision of the convex hull of the set.

Definition 13 A triangulation of a finite set $P \subset \mathbb{R}^{k}$ is a complex $\mathscr{K}$ so that the body of the complex is $\operatorname{body}(\mathscr{K})=\operatorname{conv}(P)$ and the set of vertices of the polytopes in the collection $\mathscr{K}$ is the set $P$.

In this paper, we use the Delaunay triangulation. There are different definitions for the Delaunay triangulation. Following (Edelsbrunner and Shah, 1994), we use the following definition.

Definition 14 A triangulation $\mathscr{D}$ of a set $P$ is a Delaunay triangulation if for every polytope $\mathscr{P}\left(p^{1}, \ldots, p^{a+1}\right) \in \mathscr{D}$ there exists an open ball $B$ so that $B \cap P=\emptyset$ and $\operatorname{cl}(B) \cap P=\left\{p^{1}, \ldots, p^{a+1}\right\}$.

In an informal fashion, one may say that the Delaunay triangulation contains all the polytopes defined by neighboring vectors. This is formalized by the link between the Delaunay triangulation and the Voronoi diagram (see e.g., (Edelsbrunner, 1987)).

An important concept concerning Delaunay triangulations and their construction is so-called general position of vectors (see e.g., (Rajan, 1994)). In this paper we do not assume general position, because often it is not valid for outcomes of a multiobjective optimization problem. See e.g., the first example in Section 6, where the outcomes in $P$ are not in general position.

Delaunay triangulations can be constructed with methods proposed in many papers e.g., (Edelsbrunner, 1987; Rajan, 1994). For this reason this is not a topic of this paper, but we assume that some existing method has been used to construct a Delaunay triangulation of the initial set of Pareto optimal outcomes $P$.

In this section, we have introduced the main concepts from computational geometry that are used in this paper. In the next section, we further develop these concepts for our purposes.

\section{Inherently Nondominated Polytopes and Complexes}

In this section, we discuss inherently nondominated polytopes and complexes. The definition of an inherently nondominated polytope follows the original definition of an inherently nondominated set established in Definition 1 and in Definition 15 inherent nondominance is generalized for complexes. 
According to Proposition 5, the dimension of an $a$-polytope is at most $a$. According to the following theorem, irrespective of the number of vertices, the dimension of an inherently nondominated polytope is at most the number of objectives minus one.

Theorem 1 The dimension of an inherently nondominated polytope $K \subset \mathbb{R}^{k}$ is at most $k-1$.

Proof Assume that $K=\mathscr{P}\left(z^{1}, \ldots, z^{a+1}\right)$ with $a \geq k$, because if it held that $a<k$ then the claim would be trivially true by Proposition 5 . It can be seen that $K \subset z^{1}+L$ with $L=\left\{l \in \mathbb{R}^{k}: l=\sum_{i=2}^{a+1} \lambda^{i}\left(z^{i}-z^{1}\right), \lambda^{i} \in \mathbb{R}\right.$ for all $\left.i=2, \ldots, a+1\right\}$, because every vector $s \in K$ can be written as $s=z^{1}+\sum_{i=2}^{a+1} \lambda^{i}\left(z^{i}-z^{1}\right)$ with $\lambda^{i} \in \mathbb{R}$ for all $i=2, \ldots, a+1$. Thus, it remains to prove that $\operatorname{dim}(L) \leq k-1$. To prove this assume the contrary i.e., $\operatorname{dim}(L)=k$. If $\operatorname{dim}(L)=k$, then there are linearly independent vectors $v^{1}, \ldots, v^{k} \in$ $\left\{z^{2}-z^{1}, \ldots, z^{a+1}-z^{1}\right\}$. Choose $z=\sum_{i=1}^{k}(1 / k) v^{i}$. By Propositions 7 and 3 and because the polytope $\mathscr{P}\left(v^{1}, \ldots, v^{k}\right)$ is now a simplex, it holds that $z \in \operatorname{int}\left(\mathscr{P}\left(v^{1}, \ldots, v^{k}\right)\right)$ and by Proposition 1 it holds that $\operatorname{int}\left(\mathscr{P}\left(v^{1}, \ldots, v^{k}\right)\right) \subset \operatorname{int}\left(\mathscr{P}\left(z^{2}-z^{1}, \ldots, z^{a+1}-z^{1}\right)\right)$. Therefore, $z^{1}+z \in \operatorname{int}(K)$ and there exists a real number $r>0$ so that $\mathrm{B}\left(z^{1}+z, r\right) \subset K$, where $\mathrm{B}\left(z^{1}+z, r\right)$ is a ball with radius $r$ centered at $z^{1}+z$, and, especially, it holds that $z^{1}+z-(r / 2, \ldots, r / 2) \in K$. This is a contradiction with the fact that the polytope $K$ is inherently nondominated, since it holds that $z^{1}+z-(r / 2, \ldots, r / 2)<z^{1}+z$. This completes the proof.

Corollary 1 A $k$-simplex is not inherently nondominated.

Proof This follows from Proposition 6 and Theorem 1.

An inherently nondominated polytope is always a union of polytopes with fewer vertices than objectives. This is given by the following theorem.

Theorem 2 If a polytope $K=\mathscr{P}\left(z^{1}, \ldots, z^{a+1}\right) \subset \mathbb{R}^{k}$ is inherently nondominated, then there exist $a_{i}$-polytopes $K^{i} \subset \mathbb{R}^{k}, i=1, \ldots, t$, so that $K=\cup_{i=1}^{t} K^{i}$ and $a_{i} \leq k-1$ for every $i=1, \ldots, t$.

Proof Let a vector $s \in K$ be arbitrary. By Carathéodory's theorem (Caratheodory, 1913), the vector $s$ belongs to some $k$-polytope $K^{\prime}=\mathscr{P}\left(v^{1}, \ldots, v^{k+1}\right)$ with $v^{1}, \ldots, v^{k+1} \in$ $\left\{z^{1}, \ldots, z^{a+1}\right\}$. Assume that $s=\sum_{i=1}^{k+1} \lambda^{i} v^{i} \in K$ for some $\lambda^{i} \in[0,1]$. By the proof of Theorem 1, there exists a linear subspace $L$ of $\mathbb{R}^{k}$ so that $\operatorname{dim}(L) \leq k-1$ and $K^{\prime}-v^{1} \subset$ $L$. Thus, there exist real numbers $\mu^{2}, \ldots, \mu^{k+1} \in \mathbb{R}$ so that $\sum_{i=2}^{k+1} \mu^{i}\left(v^{i}-v^{1}\right)=0$ and $\mu^{j} \neq 0$ for some $j \in\{2, \ldots, k+1\}$. Thus by choosing $\mu^{1}=-\sum_{i=2}^{k+1} \mu^{i}$, it holds that $\sum_{i=1}^{k+1} \mu^{i} v^{i}=0$ and $\sum_{i=1}^{k+1} \mu^{i}=0$. Therefore, for all $\alpha \in \mathbb{R}$ it holds that

$$
s=\sum_{i=1}^{k+1} \lambda^{i} v^{i}-\alpha \sum_{i=1}^{k+1} \mu^{i} v^{i}=\sum_{i=1}^{k+1}\left(\lambda^{i}-\alpha \mu^{i}\right) v^{i}
$$

and $\sum_{i=1}^{k+1} \lambda^{i}-\alpha \mu^{i}=1$ Especially by choosing

$$
\alpha=\min _{\substack{i=1, \ldots, k+1 \\ \mu^{i} \neq 0}} \lambda^{i} / \mu^{i}
$$


it holds in addition that $\lambda^{i}-\alpha \mu^{i} \geq 0$ for all $i=1, \ldots, k+1$ and $\lambda^{i}-\alpha \mu^{i}=0$ for some $i \in\{1, \ldots, k+1\}$. Thus, $s$ is presented as a convex combination of $k$ vectors and, thus, $s$ belongs to some $b$-polytope with $b \leq k-1$. It follows that $K \subset \cup_{i=1}^{t} K^{i}$ for some $a^{i}$ polytopes $K^{i}$ with $a^{i} \leq k-1$ for all $i=1, \ldots, t$. From the construction it is seen that we have for each polytope $K^{i}=\mathscr{P}\left(u^{1}, \ldots, u^{b+1}\right)$ with $\left\{u^{1}, \ldots, u^{b+1}\right\} \subset\left\{z^{1}, \ldots, z^{a+1}\right\}$. Thus, also $K \supset \cup_{i=1}^{t} K^{i}$ by Proposition 1 . This proves the claim.

The inherent nondominance was defined in (Hartikainen et al., 2011) for sets and not for collections of polytopes that we need in this paper. For this reason, we need to extend that definition for our needs.

Definition 15 A collection of polytopes $\mathscr{K}$ is said to be inherently nondominated if the set $\cup_{K \in \mathscr{K}} K$ is inherently nondominated. Especially, a complex is inherently nondominated if its body is inherently nondominated.

Finally, we have extensions to Definitions 2 and 3. These are the main concepts in this paper.

Definition 16 A collection of polytopes $\mathscr{K}$ is said to be an inherently nondominated Pareto front approximation if the collection of polytopes is inherently nondominated and the singleton $\{p\} \in \mathscr{K}$ for all $p \in P$.

Analogously to what is noticed in (Hartikainen et al., 2011), the complex $\{\emptyset\} \cup$ $\{\{p\}, p \in P\}$ is by definition an inherently nondominated Pareto front approximation. However, it is not a good approximation because it does include any intermediate outcomes but only the initial Pareto optimal outcomes in $P$. This is why the following definition is important. The collection $\mathscr{K}$ in the following definition is further on chosen as a Delaunay triangulation of the initial set of Pareto optimal outcomes P.

Definition 17 Let $\mathscr{K}$ be a collection of polytopes. A collection of polytopes $\mathscr{A}$ is said to be a $\mathscr{K}$-maximal inherently nondominated Pareto front approximation if the collection $\mathscr{A}$ is an inherently nondominated Pareto front approximation and for all polytopes $K \in \mathscr{K} \backslash \mathscr{A}$ the collection $\mathscr{A} \cup\{K\}$ is not inherently nondominated.

An important property is that a $\mathscr{K}$-maximal inherently nondominated Pareto front approximation is a complex whenever $\mathscr{K}$ is. This is given by the following theorem.

Theorem 3 Let $\mathscr{K}$ be a complex so that singleton $\{p\} \in \mathscr{K}$ for all $p \in P$. If a collection of polytopes $\mathscr{A}$ is a $\mathscr{K}$-maximal inherently nondominated Pareto front approximation, then the collection $\mathscr{A} \subset \mathscr{K}$ is a complex.

Proof The collection $\mathscr{A}$ is not empty, because the collection of sets $\{\emptyset\} \cup\{\{p\}: p \in$ $P\}$ is an inherently nondominated Pareto front approximation. Assume a polytope $K \in \mathscr{A}$ and let $F$ be a face of the polytope $K$. Then two observations are immediate: (i) the face $F \in \mathscr{K}$, because the collection $\mathscr{K}$ is a complex, and (ii) the collection $\mathscr{A} \cup$ $\{F\}$ is inherently nondominated, because its body is $\operatorname{body}(\mathscr{A} \cup\{F\})=\operatorname{body}(\mathscr{A})$ since by Definition 7 face $F \subset K$. Observations (i) and (ii) yield that also the face $F \in \mathscr{A}$ which implies property (1) in Definition 11. Property (2) is trivially true, because $\mathscr{A} \subset \mathscr{K}$. 
Finally, we have the following theorem implying the existence of at least one $\mathscr{K}$-maximal inherently nondominated Pareto front approximation for an appropriate complex $\mathscr{K}$. The following theorem combined with Theorem 3 yields that there exists a complex $\mathscr{A} \subset \mathscr{K}$ that is a $\mathscr{K}$-maximal inherently nondominated Pareto front approximation. In multiobjective optimization problems with more than two objectives, the maximal inherently nondominated Pareto front approximation may not be unique. For the proof of existence we need the Zorn's lemma.

Lemma 1 (Zorn's lemma) Every partially ordered set, in which every chain (i.e. totally ordered subset) has an upper bound, contains at least one maximal element.

Theorem 4 Let $\mathscr{K}$ be a complex so that $\{p\} \in \mathscr{K}$ for all $p \in P$. Then there exists a $\mathscr{K}$-maximal inherently nondominated Pareto front approximation.

Proof Define a family of collections of polytopes

$$
\mathbb{K}=\{\mathscr{B}: \mathscr{B} \subset \mathscr{K} \text { is an inherently nondominated Pareto front approximation. }\} \text {. }
$$

The collection $\{\{p\}: p \in P\} \in \mathbb{K}$ and thus $\mathbb{K}$ is not an empty family. Relation $\subset$ is a partial ordering in the family of collections $\mathbb{K}$. Let collections $\mathscr{B}^{1}, \mathscr{B}^{2}, \ldots \in \mathbb{K}$ be a totally ordered chain so that $\mathscr{B}^{i-1} \subset \mathscr{B}^{i}$ for all $i=2,3, \ldots$. Each collection $\mathscr{B}^{i}$ is then especially an inherently nondominated Pareto front approximation. Define a collection $\overline{\mathscr{B}}=\cup_{i=1}^{\infty} \mathscr{B}^{i}$. It is clear that the collection $\overline{\mathscr{B}}$ is an upper bound for the chain $\mathscr{B}^{1}, \mathscr{B}^{2} \ldots$ In order to use Zorn's lemma we prove that the collection $\overline{\mathscr{B}} \in \mathbb{K}$. Trivially, the singleton $\{p\} \in \overline{\mathscr{B}}$ for all $p \in P$, because $\{p\} \in \mathscr{B}^{1}$ by definition. Assume now that the collection $\overline{\mathscr{B}}$ is not inherently nondominated. Then there exist vectors $a^{1}, a^{2} \in \cup_{K \in \overline{\mathscr{B}}} K$ so that $a^{1} \leq a^{2}$. But then $a^{1} \in \cup_{K \in \mathscr{B}^{i}} K$ and $a^{2} \in \cup_{K \in \mathscr{B}^{i}} K$ for some $i^{1}, i^{2} \in \mathbb{N}$ and, moreover, the vectors $a^{1}, a^{2} \in \cup_{K \in \mathscr{B}}^{\max \left\{i^{1}, i^{2}\right\}} K$. This implies that the collection $\mathscr{B}^{\max \left\{i^{1}, i^{2}\right\}}$ is not inherently nondominated, which is a contradiction with the fact that the collection $\mathscr{B}^{\max \left\{i^{1}, i^{2}\right\}} \in \mathbb{K}$. Since all the assumptions of the Zorn's lemma have been fulfilled, there exists a maximal element within $\mathbb{K}$. Trivially, this maximal element is a $\mathscr{K}$-maximal inherently nondominated Pareto front approximation.

\section{The Construction of a Maximal Inherently Nondominated Pareto Front Approximation}

Naturally, the existence of a maximal inherently nondominated Pareto front approximation given by Theorem 4 is not sufficient for practical uses. In this section, we elaborate on how to construct a sub-complex of a Delaunay triangulation $\mathscr{D}$ of the initial set of Pareto optimal outcomes $P$ that is a $\mathscr{D}$-maximal inherently nondominated Pareto front approximation. Because of Corollary 1, we however exclude the $k$-simplices, with $k$ being the number of objectives of the initial multiobjective optimization problem, from the complex $\mathscr{D}$. Without confusion, we use the same symbol $\mathscr{D}$ for this sub-complex of the Delaunay triangulation. Even if Theorem 2 implies that every inherently nondominated polytope can be represented as a union of polytopes 
with the number of vertices being lower than $k$, these polytopes may not be included in the Delaunay triangulation of $P$.

We first develop methods for checking whether or not a collection of polytopes is inherently nondominated. In Subsection 5.1, we show that checking this amounts to checking all pairs of polytopes in the collection for a certain property. In Subsection 5.2, we show how to use the concepts developed in Subsection 5.1 for constructing a $\mathscr{D}$-maximal inherently nondominated Pareto front approximation.

\subsection{Checking if a Collection of Polytopes is Inherently Nondominated}

In this subsection, we show an efficient way of checking whether a collection of polytopes, or a complex, is inherently nondominated. We first define what is meant by dominance between polytopes.

Definition 18 Let $K^{1}, K^{2} \subset \mathbb{R}^{k}$ be polytopes. The polytope $K^{1}$ is said to dominate (to be dominated by) the polytope $K^{2}$, if there exist vectors $s^{1} \in K^{1}$ and $s^{2} \in K^{2}$ so that $s^{1} \leq s^{2}\left(s^{2} \leq s^{1}\right)$. If the 0-polytope $\mathscr{P}(z)=\{z\}$ dominates (is dominated by) a polytope $K \subset \mathbb{R}^{k}$ then the vector $z$ is said to dominate (be dominated by) the polytope $K$.

The connection between inherent nondominance and dominance between sets is given by the two following theorems. Theorem 5 deals with the inherent nondominance of a polytope and Theorem 6 deals with the inherent nondominance of a collection of polytopes.

Theorem 5 A polytope is inherently nondominated if and only if the polytope does not dominate itself.

Proof The proof follows directly from Definitions 1 and 18.

Theorem 6 Let $\mathscr{K}$ be a collection of polytopes. The collection $\mathscr{K}$ is inherently nondominated if and only if there does not exist polytopes $K^{1}, K^{2} \in \mathscr{K}$ so that $K^{1}$ dominates $K^{2}$.

Proof " $\Rightarrow "$ : Assume the contrary, i.e., there exist polytopes $K^{1}, K^{2} \in \mathscr{K}$ so that the polytope $K^{1}$ dominates the polytope $K^{2}$. This means that there exists a vector $s^{1} \in K^{1}$ that dominates another vector $s^{2} \in K^{2}$. This is a contradiction with the assumption that the collection of polytopes $\mathscr{K}$ is inherently nondominated, since the vectors $s^{1}, s^{2} \in \operatorname{body}(\mathscr{K})$.

$" \Leftarrow ":$ Assume that the collection of polytopes $\mathscr{K}$ is not inherently nondominated. Then there exist vectors $s^{1}, s^{2} \in \operatorname{body}(\mathscr{K})$ so that the vector $s^{1}$ dominates the vector $s^{2}$. This means that there exist polytopes $K^{1}, K^{2} \in \mathscr{K}$ so that the vector $s^{1} \in K^{1}$ and the vector $s^{2} \in K^{2}$. But then, by Definition 18, the polytope $K^{1}$ dominates the polytope $K^{2}$ which is a contradiction with the assumption.

As shown by Theorems 5 and 6, checking whether a polytope or a collection of polytopes is inherently nondominated can be done by checking for dominance between polytopes as defined in Definition 18. For this reason we need an efficient way 
of checking for dominance between polytopes. According to the following theorem, the dominance between polytopes can be checked by solving optimization problems.

Theorem 7 Let $K^{1}, K^{2} \subset \mathbb{R}^{k}$ be non-empty polytopes. Consider optimization problems

$$
\min _{\text {s.t. } s^{1} \in K^{1}, s^{2} \in K^{2}} \max _{i=1, \ldots, k}\left(s_{i}^{1}-s_{i}^{2}\right)
$$

and

$$
\begin{array}{ll}
\min & \sum_{i=1}^{k}\left(s_{i}^{1}-s_{i}^{2}\right) \\
\text { s.t. } & s^{1} \in K^{1}, s^{2} \in K^{2} \\
& s_{i}^{1} \leq s_{i}^{2} \text { for all } i=1, \ldots, k .
\end{array}
$$

The polytope $K^{1}$ dominates the polytope $K^{2}$ if and only if one of the following holds:

(i) the optimal value in problem (2) is less than zero

OR

(ii) the optimal value in problem (2) is exactly zero and the optimal value in problem (3) is less than zero.

Proof Notice that problem (2) has a solution, because the outer objective function $g\left(s^{1}, s^{2}\right):=\max _{i=1, \ldots, k}\left(s_{i}^{1}-s_{i}^{2}\right)$ is continuous and the feasible set is compact and nonempty. Furthermore, problem (3) has a solution, if the optimal value in problem (2) is zero, because the objective function is continuous and the feasible set is compact and non-empty.

By Definition 18, if the polytope $K^{1}$ dominates the polytope $K^{2}$ then there exist vectors $s^{1} \in K^{1}$ and $s^{2} \in K^{2}$ so that $s^{1}$ dominates $s^{2}$. The dominance can either be strong or weak i.e., either

(1) $s_{i}^{1}<s_{i}^{2}$ for all $i=1, \ldots, k$

OR

(2) $s_{i}^{1} \leq s_{i}^{2}$ for all $i=1, \ldots, k$ and $s_{j}^{1}<s_{j}^{2}$ for some index $j \in\{1, \ldots, k\}$.

Clearly, alternative $(i)$ holds if and only if alternative (1) holds. Assume now that alternative (2) holds but alternative $(i)$ does not hold. Then the optimal value in problem (2) is equal to zero, because if it was more than zero alternative (2) could not hold, and the optimal value in problem (3) is less than zero, because $\sum_{i=1}^{k}\left(s_{i}^{1}-s_{i}^{2}\right)<$ $s_{j}^{1}-s_{j}^{2}$ for all $j \in\{1, \ldots, k\}$. Finally, if alternative (ii) holds, then there exist vectors $s^{1} \in K^{1}$ and $s^{2} \in K^{2}$ so that $s_{i}^{1} \leq s_{i}^{2}$ for all $i=1, \ldots, k$ and $\sum_{i=1}^{k}\left(s_{i}^{1}-s_{i}^{2}\right)<0$. But if $\sum_{i=1}^{k}\left(s_{i}^{1}-s_{i}^{2}\right)<0$, then there exists an index $j \in\{1, \ldots, k\}$ so that $s_{j}^{1}<s_{j}^{2}$ and thus alternative (2) must hold. This completes the proof.

\subsection{Removal of Polytopes from the Delaunay Triangulation}

Assume that we have constructed the Delaunay triangulation $\mathscr{D}$ of the initial set of Pareto optimal outcomes $P \subset \mathbb{R}^{k}$ and that we have excluded the $k$-simplices from it. In this subsection, we propose an approach to finding a collection of polytopes $\mathscr{R} \subset \mathscr{D}$ so that the collection of polytopes

$$
\mathscr{D} \backslash \mathscr{R}
$$


is a $\mathscr{D}$-maximal inherently nondominated Pareto front approximation based on the initial set of Pareto optimal outcomes $P$. In other words, we propose ways to remove polytopes from the Delaunay triangulation to get the approximation.

Without loss of generality we may assume that for all $a=0, \ldots, m-1$, the $a$ polytopes in the complex $\mathscr{D}$ are $K^{a, 1}, \ldots, K^{a, t^{a}}$. For all $a=m-1, m-2, \ldots, 1$ and for all $j=1, \ldots, t^{a}$, it holds that the polytope $K^{a, j} \in \mathscr{R}$ i.e., the polytope is removed, if either

(R1) there exists an outcome $p \in P$ that dominates or is dominated by the polytope $K^{a, j}$ or the polytope $K^{a, j}$ dominates itself

OR

(R2) there exists a $b$-polytope $K^{b, j^{\prime}} \in \mathscr{D} \backslash \mathscr{R}$ with either $b>a$ or $b=a$ and $j^{\prime}<j$ that dominates or is dominated by the polytope $K^{a, j}$.

Rule (R1) dictates that a polytope is removed if it either dominates or is dominated by some of the Pareto optimal outcomes in $P$ or if the polytope is not inherently nondominated. There may be multiple $\mathscr{D}$-maximal inherently nondominated Pareto front approximations that can be constructed from polytopes that are not removed by rule (R1) and rule (R2) dictates which of them we choose. According to it, the polytopes with more vertices are less likely to be removed from the collection because whenever we choose not to remove a polytope, then the polytopes with less vertices are removed if they dominate or are dominated by this polytope.

Theorem 8 If the collection $\mathscr{R}$ is given by rules $(R 1)$ and $(R 2)$ then the collection of polytopes given by (4) is a $\mathscr{D}$-maximal inherently nondominated Pareto front approximation.

Proof The polytopes in the complex $\mathscr{D}$ can be ordered as in rule (R2) with respect to the number of vertices $a$ and the index $j$. According to rule (R2) a polytope with at least two vertices is removed from the complex if there exists a polytope prescribed by this rule that is either dominated or dominates this polytope. For this reason there cannot be distinct polytopes $K^{i}, K^{j} \in \mathscr{D} \backslash \mathscr{R}$ with more vertices than 2 that dominate each other. The first part of rule (R1) dictates that there cannot be a polytope in the collection that dominates or is dominated by an outcome in $P$ and, thus, there are no distinct polytopes in the collection that dominate each other. According to the second part of rule (R1), no polytope in $\mathscr{D} \backslash \mathscr{R}$ is dominated by it self. According to Theorem 7, the collection of polytopes $\mathscr{D} \backslash \mathscr{R}$ is inherently nondominated and since the singleton $\{p\} \in \mathscr{D} \backslash \mathscr{R}$ for all $p \in P$, the collection of polytopes is an inherently nondominated Pareto front approximation. Trivially, the Pareto front approximation is $\mathscr{D}$-maximal.

According to (McMullen, 1970), a Delaunay triangulation of the set $P$ contains at most $O\left(m^{\lceil k / 2\rceil}\right)$ polytopes, where $m$ is the number of outcomes in $P$ and $k$ is the number of objectives of the multiobjective optimization problem. For this reason, one may have to solve $O\left(\mathrm{~m}^{k}\right)$ optimization problems in order to find out all dominations between the polytopes in the triangulation with problems (2) and (3). This is computationally the most expensive part of the approximation approach. Methods for reducing this computational expense go beyond the scope of this paper. 


\section{Examples of Inherently Nondominated Pareto Front Approximations}

In this section, we present two examples of inherently nondominated Pareto front approximations constructed as proposed in the previous section. In the first example, we approximate the Pareto front of the three-objective DTLZ5 test problem introduced in (Deb et al., 2002). The DTLZ5 test problem's special feature is that even if it has three objectives, the Pareto front is merely one-dimensional. Additionally, the outcome set $f(S)$ of the DTLZ5 test problem is nonconvex. For these reasons, approximating the Pareto front of this problem is not trivial, which makes it a suitable task for demonstrating the power of our approximation approach. Even though the DTLZ5 problem is not computationally expensive, we can demonstrate our approximation approach with it, because the computational expense of the problem only affects the computation of the Pareto optimal outcomes $P$ and not the Pareto front approximation. The second example concerns approximating the Pareto front of the three-objective DTLZ2 test problem (Deb et al., 2002). In this example, we do not show the construction of the approximation but merely give the inherently nondominated Pareto front approximation to further illustrate the versatility of our approach.

The DTLZ5 test problem can be formulated as

$$
\begin{array}{ll}
\min & \left(f_{1}(x), f_{2}(x), f_{3}(x)\right) \\
\text { s.t. } & 0 \leq x_{i} \leq 1 \text { for all } i=1,2,3 \\
\text { where } & f_{1}(x)=\left(1+x_{3}^{0.1}\right) \cos \left(\frac{x_{1} \pi}{2}\right) \cos (\theta(x)) \\
& f_{2}(x)=\left(1+x_{3}^{0.1}\right) \cos \left(\frac{x_{1} \pi}{2}\right) \sin (\theta(x)) \\
& f_{3}(x)=\left(1+x_{3}^{0.1}\right) \sin \left(\frac{x_{1} \pi}{2}\right) \\
& \theta(x)=\frac{\pi}{4\left(1+x_{3}^{0.1}\right)}\left(1+2 x_{3}^{0.1} x_{2}\right) .
\end{array}
$$

The actual Pareto front of the DTLZ5 test problem is explicitly known and given as

$$
\left\{\left(f_{1}(x), f_{2}(x), f_{3}(x)\right)^{T}: x_{3}=0\right\} .
$$

Assume that the set of initial Pareto optimal outcomes is

$$
\begin{aligned}
P & =\left\{p^{1}, p^{2}, p^{3}, p^{4}, p^{5}\right\} \\
& =\left\{\left(\frac{1}{\sqrt{2}}, \frac{1}{\sqrt{2}}, 0\right),\left(\frac{\sqrt{2+\sqrt{2}}}{2 \sqrt{2}}, \frac{\sqrt{2+\sqrt{2}}}{2 \sqrt{2}}, \frac{\sqrt{2-\sqrt{2}}}{2}\right),\right. \\
& \left.\left(\frac{1}{2}, \frac{1}{2}, \frac{1}{\sqrt{2}}\right),\left(\frac{\sqrt{2-\sqrt{2}}}{2 \sqrt{2}}, \frac{\sqrt{2-\sqrt{2}}}{2 \sqrt{2}}, \frac{\sqrt{2+\sqrt{2}}}{2}\right),(0,0,1)\right\}
\end{aligned}
$$

given by $x \in\left\{\left(x_{1}, x_{2}, x_{3}\right): x_{1}=0,1 / 4,1 / 2,3 / 4,1, x_{2}=0\right.$ and $\left.x_{3}=0\right\}$.

A Delaunay triangulation of this set is given by

$$
\begin{aligned}
\mathscr{D}= & \left\{\emptyset, \mathscr{P}\left(p^{1}\right), \mathscr{P}\left(p^{2}\right), \mathscr{P}\left(p^{3}\right), \mathscr{P}\left(p^{4}\right), \mathscr{P}\left(p^{5}\right), \mathscr{P}\left(p^{1}, p^{2}\right), \mathscr{P}\left(p^{2}, p^{3}\right),\right. \\
& \left.\mathscr{P}\left(p^{3}, p^{4}\right), \mathscr{P}\left(p^{4}, p^{5}\right), \mathscr{P}\left(p^{5}, p^{1}\right), \mathscr{P}\left(p^{1}, p^{2}, p^{3}, p^{4}, p^{5}\right)\right\}, \\
= & \left\{K^{1}, \ldots, K^{12}\right\} .
\end{aligned}
$$


The dimension of polytope $K^{12}$ is two so it is not removed before applying the rules. The different dominations between polytopes in the Delaunay triangulation can be presented in a matrix with entries $a_{i j}$ equal to 1 if the polytope $K^{i}$ dominates the polytope $K^{j}$ and equal to 0 otherwise. In this problem, the dominations yield a $12 \times 12$ matrix

$$
\left[\begin{array}{cccccccccccc}
0 & 0 & 0 & 0 & 0 & 0 & 0 & 0 & 0 & 0 & 0 & 0 \\
\vdots & \vdots & \vdots & \vdots & \vdots & \vdots & \vdots & \vdots & \vdots & \vdots & \vdots & \vdots \\
0 & 0 & 0 & 0 & 0 & 0 & 0 & 0 & 0 & 0 & 0 & 0 \\
0 & 0 & 1 & 1 & 1 & 0 & 1 & 1 & 1 & 1 & 0 & 1 \\
0 & 0 & 1 & 1 & 1 & 0 & 1 & 1 & 1 & 1 & 0 & 1
\end{array}\right],
$$

where all entries in the 10 top rows are zeros. As an example we verify that polytope $K^{7}=\mathscr{P}\left(p^{1}, p^{2}\right)$ is dominated by polytope $K^{11}=\mathscr{P}\left(p^{5}, p^{1}\right)$ using problems (2) and (3). Problem (2) becomes

$$
\begin{array}{ll}
\min & \max _{i=1, \ldots, k}\left(s_{i}^{1}-s_{i}^{2}\right) \\
\text { s.t. } & s^{1}=\left(1-\lambda^{1}\right)\left(\frac{1}{\sqrt{2}}, \frac{1}{\sqrt{2}}, 0\right)+\lambda^{1}(0,0,1) \\
& s^{2}=\left(1-\lambda^{2}\right)\left(\frac{1}{\sqrt{2}}, \frac{1}{\sqrt{2}}, 0\right)+\lambda^{2}\left(\frac{\sqrt{2+\sqrt{2}}}{2 \sqrt{2}}, \frac{\sqrt{2+\sqrt{2}}}{2 \sqrt{2}}, \frac{\sqrt{2-\sqrt{2}}}{2}\right) \\
& 0 \leq \lambda^{j} \leq 1 \text { for } j=1,2
\end{array}
$$

and with some reformulation it assumes the form of a linear optimization problem

$$
\begin{array}{ll}
\min t & \\
\text { s.t. } & t \geq \frac{2-\sqrt{2+\sqrt{2}}}{2 \sqrt{2}} \lambda^{2}-\frac{1}{\sqrt{2}} \lambda^{1} \\
& t \geq \lambda^{1}-\frac{\sqrt{2-\sqrt{2}}}{2} \lambda^{2} \\
& 0 \leq \lambda^{j} \leq 1 \text { for } j=1,2 .
\end{array}
$$

This problem can be solved with e.g., the simplex method and the optimal value of $t$ is

$$
\frac{2-\sqrt{2+\sqrt{2}}-\sqrt{2-\sqrt{2}}}{2(\sqrt{2}+1)} \approx-0.12698(<0) .
$$

By Theorem 7 , the polytope $\mathscr{P}\left(p^{5}, p^{1}\right)$ dominates polytope $\mathscr{P}\left(p^{1}, p^{2}\right)$.

Notice that in the complex $\mathscr{D}$ the polytope $\emptyset$ is a -1 -polytope, the polytopes $K^{2}, \ldots, K^{6}$ are 0 -polytopes, the polytopes $K^{7}, \ldots, K^{11}$ are 1 -polytopes and the polytope $K^{12}$ is a 4-polytope. The polytope $K^{12} \in \mathscr{R}$ by rule (R1), because it dominates itself. This shows up in the above matrix with the entry $a_{12,12}=1$. Of the 1-polytopes, the polytope $K^{11}$ dominates e.g., the outcome $p^{3} \in P$ and, thus, $K^{11} \in \mathscr{R}$ by rule (R1). This rule does not apply to the other polytopes except for $K^{11}$ and $K^{12}$. As it can be seen from the matrix, there is no domination left when the polytopes $K^{11}$ and $K^{12}$ have been removed from the collection. For this reason, rule (R2) does not apply to any of the polytopes $K^{1}, \ldots, K^{10}$. Thus, the $\mathscr{D}$-maximal inherently nondominated Pareto front approximation is

$$
\begin{aligned}
\mathscr{D} \backslash \mathscr{R} & =\mathscr{D} \backslash\left\{\mathscr{P}\left(p^{1}, p^{2}, p^{3}, p^{4}, p^{5}\right), \mathscr{P}\left(p^{1}, p^{5}\right)\right\} . \\
& =\left\{K^{1}, \ldots, K^{10}\right\}
\end{aligned}
$$




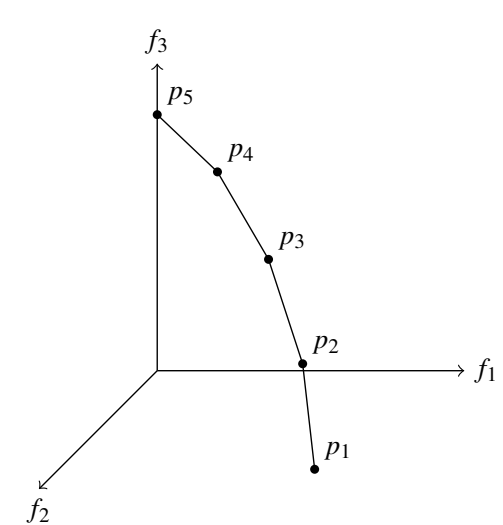

(a) A $\mathscr{D}$-maximal inherently nondominated Pareto front approximation of the DTLZ5 test problem based on 5 Pareto optimal outcomes.

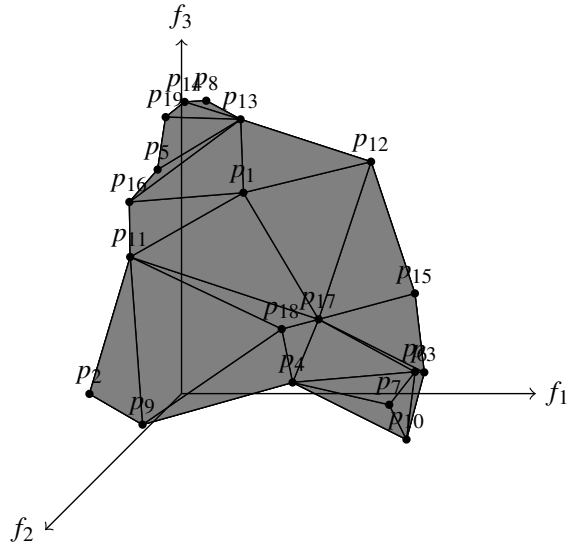

(b) An inherently nondominated Pareto front approximation of the DTLZ2 test problem based on 19 Pareto optimal outcomes.

Fig. 1: Bodies of two inherently nondominated Pareto front approximations

The body of this complex is shown in Figure 1a.

The same approach applied to the DTLZ2 test problem introduced in (Deb et al., 2002) with 19 random Pareto optimal outcomes $p_{1}, \ldots, p_{19}$ yields the approximation shown in Figure 1b. As in the DTLZ5 problem, the Pareto front of this problem is not convex, either.

The two examples presented in this section demonstrate that the proposed approximation approach can handle unusual or difficult problems. The Pareto front of the DTLZ5 test problem is merely one-dimensional while the problem has three objectives and the DTLZ2 test problem is nonconvex. In both cases, the approach produces an interpolation between the known Pareto optimal outcomes that can be used with an interactive method through the surrogate problem defined in Hartikainen et al. (2011).

\section{Two Special Cases of Multiobjective Optimization Problems}

As in the previous section, assume that $\mathscr{D}$ is a Delaunay triangulation of the initial set of Pareto optimal outcomes $P$ and that the $k$-simplices have been excluded from it. In this section, we demonstrate how the concept of a $\mathscr{D}$-maximal inherently nondominated Pareto front approximation applies to two special cases, which are the biobjective case and the $\mathbb{R}_{+}^{k}$-convex case. It is shown that in these special cases the approximation is similar to some approximations in literature and, thus, the approach proposed in this paper can be seen as a generalization of those methods. 


\subsection{Biobjective Case}

If the number of objectives of the multiobjective optimization problem is two, then the $\mathscr{D}$-maximal inherently nondominated Pareto front approximation is unique and can be explicitly stated. With the help of the following theorem one may construct a $\mathscr{D}$-maximal inherently nondominated Pareto front approximation without the rules (R1) and (R2).

Theorem 9 If the number of objectives is $k=2$ and the initial set of Pareto optimal outcomes is $P=\left\{p^{1}, \ldots, p^{m}\right\}$ so that $p_{1}^{1}<p_{1}^{2}<\ldots<p_{1}^{m}$, then the only $\mathscr{D}$-maximal inherently nondominated Pareto front approximation is

$$
\mathscr{K}=\{\emptyset\} \cup\{\{p\}: p \in P\} \cup\left\{\mathscr{P}\left(p^{i-1}, p^{i}\right): i=2, \ldots, m\right\} .
$$

Proof Notice that since the outcomes in $P$ are Pareto optimal, it must be that $p_{2}^{1}>$ $p_{2}^{2}>\ldots>p_{2}^{m}$. Thus, the complex $\mathscr{K}$ is inherently nondominated, because if $s^{1}, s^{2} \in$ $\operatorname{body}(\mathscr{K})$ and $s_{1}^{1}<s_{1}^{2}$ then $s_{2}^{1}>s_{2}^{2}$.

Assume now a non-empty polytope $K \in \mathscr{D}$ so that $\mathscr{K} \cup\{K\}$ is inherently nondominated and let a vector $s \in K$ be arbitrary. Since $s \in \operatorname{conv}(P)$, it must be that $p_{1}^{1} \leq s_{1} \leq p_{1}^{m}$. Thus, there must be a vector $s^{\prime} \in \operatorname{body}(\mathscr{K})$ so that $s_{1}=s_{1}^{\prime}$. Because the complex $\mathscr{K} \cup\{K\}$ is assumed inherently nondominated, it must be that $s_{2}^{\prime}=s_{2}$ and, thus, $s^{\prime}=s$. Therefore, $K \subset \operatorname{body}(\mathscr{K})$. Because the collection $\mathscr{D}$ is a sub-complex of a Delaunay triangulation of the set $P$, it must be that $K=K^{\prime}$ for some $K^{\prime} \in \mathscr{K}$. Thus, the complex $\mathscr{K}$ is a $\mathscr{D}$-maximal inherently nondominated Pareto front approximation.

For uniqueness of the complex $\mathscr{K}$, assume another inherently nondominated Pareto front approximation $\mathscr{K}^{\prime} \subset \mathscr{D}$ so that $\mathscr{K}^{\prime} \backslash \mathscr{K} \neq \emptyset$. Choose a non-empty polytope $K^{\prime} \in \mathscr{K}^{\prime} \backslash \mathscr{K}$. Since $\operatorname{vert}\left(K^{\prime}\right) \subset P, \mathscr{K}^{\prime}$ is a complex and $K^{\prime} \notin \mathscr{K}$, there must be $i, j \in\{1, \ldots, m\}$ so that $j-i>1$ and $\mathscr{P}\left(p^{i}, p^{j}\right) \subset K^{\prime}$ and $p^{i+1} \notin \mathscr{P}\left(p^{i}, p^{j}\right)$. However, by Definition $16\left\{p^{i+1}\right\} \in \mathscr{K}^{\prime}$ and since $\mathscr{K}^{\prime}$ is inherently nondominated, $p^{i+1} \in \mathscr{P}\left(p^{i}, p^{j}\right)$.

Corollary 2 If the number of objectives is $k=2$, then the $\mathscr{D}$-maximal inherently nondominated Pareto front approximation is connected.

According to Theorem 9, the only $\mathscr{D}$-maximal inherently nondominated Pareto front approximation is the piece-wise linear curve that connects adjacent Pareto optimal outcomes. This approximation has been used in e.g., in (Schandl et al., 2001).

\subsection{Convex Case}

In this subsection, we study the structure of the Pareto front approximation in convex multiobjective optimization problems. Convex multiobjective optimization problems have been studied extensively (see e.g., (Ruzika and Wiecek, 2005)). For our treatment of this case, we need a lemma from (Yu and Zeleny, 1975). 
Lemma 2 The Pareto front of a polytope can be represented as a union of the Pareto faces (i.e., the faces of the polytope in which all the vectors are Pareto optimal) of the polytope.

Definition 19 Assume that $B \subset \mathbb{R}^{k}$ is a set. Then a set $A \subset \mathbb{R}^{k}$ is called $B$-convex, if the set $A+B=\{a+b: a \in A, b \in B\}$ is convex.

Theorem 10 If the outcome set $f(S)$ is $\mathbb{R}_{+}^{k}$-convex, then the complex

$$
\mathscr{K}=\{K \in \mathscr{D}: K \subset \mathrm{PF}(\operatorname{conv}(P))\}
$$

is a $\mathscr{D}$-maximal inherently nondominated Pareto front approximation.

Proof First, the collection $\mathscr{K}$ is inherently nondominated because, as noticed in (Hartikainen et al., 2011), the Pareto front is inherently nondominated and body $(\mathscr{K}) \subset$ $\operatorname{PF}(\operatorname{conv}(P))$ by definition. Second, because a face of a polytope is a subset of the polytope, then if the polytope is in the collection $\mathscr{K}$, then the face is in the collection. This again implies that the collection $\mathscr{K}$ is a complex. Thus the collection $\mathscr{K}$ is an inherently nondominated complex.

To prove that the inherently nondominated complex $\mathscr{K}$ is $\mathscr{D}$-maximal, let a polytope $K \in \mathscr{D}$ be so that the collection $\mathscr{K} \cup\{K\}$ is inherently nondominated. If $K \notin \mathscr{K}$, then there exists a vector $s \in K \backslash \mathrm{PF}(\operatorname{conv}(P))$. This immediately implies that there exists $s^{\prime} \in \operatorname{PF}(\operatorname{conv}(P))$ so that $s^{\prime} \leq s$. From Lemma 2 and Definition 13 it follows that

$$
\operatorname{PF}(\operatorname{conv}(P))=\operatorname{body}(\mathscr{K})
$$

and, thus, $s^{\prime} \in \operatorname{body}(\mathscr{K})$, which contradicts the inherent nondominance of $\mathscr{K} \cup\{K\}$. Therefore $K \in \mathscr{K}$ and the complex $\mathscr{K}$ is a $\mathscr{D}$-maximal inherently nondominated Pareto front approximation.

The set $\operatorname{PF}(\operatorname{conv}(P))$ has been used to approximate the Pareto front in e.g., (Eskelinen et al., 2010; Lotov et al., 2004). According to the following corollary the body of a $\mathscr{D}$-maximal Pareto front approximation based on $P$ is equal to this set in the $\mathbb{R}_{+}^{k}$-convex case.

Corollary 3 If the outcome set $f(S)$ is $\mathbb{R}_{+}^{k}$-convex, then there exists a $\mathscr{D}$-maximal inherently nondominated Pareto front approximation $\mathscr{K}$ so that

$$
\operatorname{body}(\mathscr{K})=\operatorname{PF}(\operatorname{conv}(P))
$$

\section{Error Estimates}

In this section, we examine how well a $\mathscr{D}$-maximal inherently nondominated Pareto front approximation $\mathscr{K}$ approximates the Pareto front. More accurately, we develop the following estimate: given a vector $s \in \operatorname{body}(\mathscr{K})$ we estimate an error vector $d(s) \in \mathbb{R}^{k}$ that fulfills two conditions:

1. there exists an outcome $z \in f(S)$ so that $z_{i} \leq s_{i}+d_{i}(s)$ for all $i=1, \ldots, k$ and

2. there does not exist an outcome $z \in f(S)$ so that $z \leq s-d(s)$. 
These error estimates should have a clear meaning to the decision maker: (1) implies that there exists an actual Pareto optimal outcome that is at least as good as $s+d(s)$ in all objectives and (2) implies that there is no actual outcome that dominates $s-d(s)$.

We estimate $d(s)$ in two ways. In Subsection 8.1, we develop estimates for $d(s)$ that require computing the approximation before the estimation and, in Subsection 8.2 , we develop estimates that can be computed by means of just the set of initial Pareto optimal outcomes $P$. The former error estimates can be used to help the decision maker in choosing a vector on the Pareto front approximation to be projected on the actual Pareto front. The latter estimates can be used for deciding whether to add more outcomes into $P$ before computing the approximation - if these error estimates are big it may be useful to compute more Pareto optimal outcomes if possible.

\subsection{Estimates after Computing the Approximation}

In this section, we assume that the complex $\mathscr{K}$ has already been computed. We develop estimates for $d(s)$ defined above. We start with a lemma.

Lemma 3 Let $s, e \in \mathbb{R}^{k}$. If there exists an outcome $p \in P$ so that $p_{i} \geq s_{i}-e_{i}$ for all $i=1, \ldots, k$, then there does not exist an outcome $z \in f(S)$ so that $z \leq s-e$.

Proof This is clear since $p$ is assumed to be Pareto optimal.

The following lemma gives the required estimates and the main result is given by Theorem 11.

Lemma 4 Let $K=\mathscr{P}\left(z^{1}, \ldots, z^{a+1}\right) \in \mathscr{K}$ be a polytope. Furthermore, let $s=\sum_{j=1}^{a+1} \lambda^{j} z^{j} \in$ $K$ be a vector in this polytope with $\lambda^{j} \geq 0$ for all $j=1, \ldots, k$ and $\sum_{j=1}^{a+1} \lambda^{j}=1$ and let $z^{\max }, z^{\min }$ and $z^{\text {dif }}$ be vectors in $\mathbb{R}^{k}$ so that for all $i \in\{1, \ldots, k\}$

$$
z_{i}^{\max }=\max _{j \in\{1, \ldots, a+1\}} z_{i}^{j}, z_{i}^{\min }=\min _{j \in\{1, \ldots, a+1\}} z_{i}^{j} \text { and } z_{i}^{\mathrm{dif}}=z_{i}^{\max }-z_{i}^{\min } .
$$

Then,

1. $s_{i}-\left(1-\lambda^{j}\right) z_{i}^{\text {dif }} \leq z_{i}^{j}$ and

2. $s_{i}+\left(1-\lambda^{j}\right) z_{i}^{\mathrm{dif}} \geq z_{i}^{j}$

for all $j=1, \ldots, a+1$ and $i=1, \ldots, k$. 
Proof Let $\hat{j} \in\{1, \ldots, a+1\}$ and $i \in\{1, \ldots, k\}$ be arbitrary. With the above definitions

$$
\begin{aligned}
s_{i}-\left(1-\lambda^{\hat{j}}\right) z_{i}^{\mathrm{dif}} & =\sum_{j=1}^{a+1} \lambda^{j} z_{i}^{j}-\left(1-\lambda^{\hat{j}}\right) z_{i}^{\mathrm{dif}} \\
& =z_{i}^{\max }+\sum_{j=1}^{a+1} \lambda^{j}\left(z_{i}^{j}-z_{i}^{\max }\right)-\left(1-\lambda^{\hat{j}}\right) z_{i}^{\mathrm{dif}} \\
& \leq z_{i}^{\max }+\lambda^{\hat{j}}\left(z_{i}^{\hat{j}}-z_{i}^{\max }\right)-\left(1-\lambda^{\hat{j}}\right) z_{i}^{\mathrm{dif}} \\
& =\left(1-\lambda^{\hat{j}}\right) z_{i}^{\max }+\lambda^{\hat{j}} z_{i}^{\hat{j}}-\left(1-\lambda^{\hat{j}}\right) z_{i}^{\max }+\left(1-\lambda^{\hat{j}}\right) z_{i}^{\min } \\
& =\lambda^{\hat{j}} z_{i}^{\hat{j}}+\left(1-\lambda^{\hat{j}}\right) z_{i}^{\min } \\
& \leq z_{i}^{\hat{j}},
\end{aligned}
$$

where the first inequality follows from the property $z_{i}^{j}-z_{i}^{\max } \leq 0$ for all $j=1, \ldots, a+$ 1 and the second one follows from the inequality $z_{i}^{\min } \leq z_{i}^{\hat{j}}$. In a similar way we obtain

$$
s_{i}+\left(1-\lambda^{\hat{j}}\right) z_{i}^{\text {dif }} \geq z_{i}^{\hat{j}} .
$$

This proves the assertion.

Theorem 11 Let $K=\mathscr{P}\left(z^{1}, \ldots, z^{a+1}\right) \in \mathscr{K}$ be a polytope. Furthermore, let $s=$ $\sum_{j=1}^{a+1} \lambda^{j} z^{j} \in K$ be a vector in this polytope and let $z^{\max }, z^{\min }$ and $z^{\mathrm{dif}}$ be defined as above. Then $d(s) \leq\left(1-\lambda^{j}\right) z^{\text {dif }}$ for all $j=1, \ldots, a+1$.

Proof This follows from Lemmas 3 and 4.

Corollary 4 With the definitions of Theorem 11, it holds that $d(s) \leq a z^{\mathrm{dif}} /(a+1)$.

Proof Since $\sum_{i=1}^{a+1} \lambda^{j}=1$, then there exists $\hat{j} \in\{1, \ldots, a+1\}$ so that $\lambda^{\hat{j}} \geq 1 /(a+1)$ and, thus, $(1-\lambda \hat{j}) \leq 1-1 /(a+1)=a /(a+1)$. This combined with Theorem 11 proves the assertion.

Example 2 Continuing the DTLZ5 example from Section 6 assume that

$$
s=1 / 3 p^{3}+2 / 3 p^{4} \approx(0.34707,0.34707,0.85162) \in K^{9}=\mathscr{P}\left(p^{3}, p^{4}\right) .
$$

Then $\left.z^{\max }=(1 / 2,1 / 2, \sqrt{2+\sqrt{2}} / 2), z^{\min }=(2-\sqrt{2}) /(2 \sqrt{2}),(2-\sqrt{2}) /(2 \sqrt{2}), 1 / \sqrt{2}\right)$ and

$$
\begin{aligned}
z^{\mathrm{dif}} & =z^{\max }-z^{\min }=\left(\frac{\sqrt{2}-\sqrt{2-\sqrt{2}}}{2 \sqrt{2}}, \frac{\sqrt{2}-\sqrt{2-\sqrt{2}}}{2 \sqrt{2}}, \frac{\sqrt{2} \sqrt{2+\sqrt{2}}-1}{2 \sqrt{2}}\right) \\
& \approx(0.22940,0.22940,0.21677) .
\end{aligned}
$$

Theorem 11 yields

$$
d(s) \leq(1-2 / 3)(0.22940,0.22940,0.21677) \approx(0.076467,0.076467,0.072257)
$$

and, thus, 
1. there exists an outcome $z \in f(S)$ that is at least as good as

$$
s+d \approx(0.42353,0.42353,0.92388)
$$

in all objectives and

2. there does not exist an outcome $z \in f(S)$ so that

$$
z \leq s-d \approx(0.27060,0.27060,0.77936) \text {. }
$$

\subsection{Estimates before Computing the Approximation}

In this section, we assume that the complex $\mathscr{K}$ has not yet been computed but we have the set of initial Pareto optimal outcomes $P$. Theorem 12 establishes an estimate for $d(s)$ without computing the complex $\mathscr{K}$.

Theorem 12 Assume that $K^{l}=\mathscr{P}\left(p^{j_{l}^{1}}, p^{j_{l}^{2}}\right)$ with Pareto optimal outcomes $p^{j_{l}^{1}}, p^{j_{l}^{2}} \in$ $P$ and $l=1, \ldots, r$ are all the 1-polytopes so that $K^{l} \cup P$ is inherently nondominated. Define a vector $e \in \mathbb{R}^{k}$ so that

$$
e_{i}=\max _{l=1, \ldots, r}\left|z_{i}^{j_{l}^{1}}-z_{i}^{j_{l}^{1}}\right|
$$

for all $i=1, \ldots, k$. Then it holds that

$$
d(s) \leq \frac{k-1}{k} e
$$

for all $s \in \operatorname{body}(\mathscr{K})$.

Proof Assume that $\mathscr{K}$ is a $\mathscr{D}$-maximal inherently nondominated Pareto front approximation. The structure of $\mathscr{K}$ remains unknown but the existence of the approximation is proven in Theorem 4 . Let a vector $s \in \operatorname{body}(\mathscr{K})$ be arbitrary. Then $s \in \mathscr{P}\left(\hat{p}^{1}, \ldots, \hat{p}^{a+1}\right)$ for some $\hat{p}^{1}, \ldots, \hat{p}^{a+1} \in P$. By Theorem 2 , there exist $z^{1}, \ldots, z^{k} \in$ $\left\{\hat{p}^{1}, \ldots, \hat{p}^{m}\right\}$ so that $s \in \mathscr{P}\left(z^{1}, \ldots, z^{k}\right)$. Now the union $\mathscr{P}\left(z^{j^{1}}, z^{j^{2}}\right) \cup P$ is inherently nondominated for all indices $j^{1}, j^{2} \in\{1, \ldots, k\}$, because $\mathscr{K}$ is an inherently nondominated Pareto front approximation and, thus,

$$
\left|z_{i}^{j^{1}}-z_{i}^{j^{2}}\right| \leq e_{i}
$$

for all indices $i, j^{1}, j^{2} \in\{1, \ldots, k\}$. This immediately implies $z_{i}^{\mathrm{dif}} \leq e_{i}$ for all $i=$ $1, \ldots, k$ with the definitions of Lemma 4 and this combined with Theorem 4 yields the claim.

In order to be useful, the computation of the above a priori error estimate should be less expensive than the computation of the whole $\mathscr{D}$-maximal inherently nondominated Pareto front approximation. This indeed is the case, if the number of objectives $k$ is sufficiently large. To find the polytopes $K^{l}$ in Theorem 12, one has to solve problems (2) and (3) $O\left(\mathrm{~m}^{3}\right)$ times, while the computation of the $\mathscr{D}$-maximal inherently nondominated Pareto front approximation demands solving problems (2) and (3) $O\left(\mathrm{~m}^{k}\right)$ times in the worst case scenario. Thus, if $k>3$, then the computation of the a priori estimate is worthwhile. 
Example 3 Continuing with the DTLZ5 example from Section 6, the 1-polytopes $K^{l}$ of Theorem 12 are $K^{7}, K^{8}, K^{9}, K^{10}$. We calculate $e_{i}=\max \left\{\left|p_{i}^{1}-p_{i}^{2}\right|,\left|p_{i}^{2}-p_{i}^{3}\right|\right.$, $\left.\left|p_{i}^{3}-p_{i}^{4}\right|,\left|p_{i}^{4}-p_{i}^{5}\right|\right\}$ for all $i=1,2,3$ and obtain

$$
\begin{aligned}
e & =\left(\frac{\sqrt{2-\sqrt{2}}}{2 \sqrt{2}}, \frac{\sqrt{2-\sqrt{2}}}{2 \sqrt{2}}, \frac{\sqrt{2-\sqrt{2}}}{2}\right) \\
& \approx(0.27060,0.27060,0.38268) .
\end{aligned}
$$

By Theorem $12, d(s) \leq 2 / 3 e \approx(0.18040,0.18040,0.25512)$ and for every $s \in \operatorname{body}(\mathscr{K})$

1. there exists an outcome $z \in f(S)$ that is at least as good as

$$
s+(0.18040,0.18040,0.25512)
$$

in all objectives and

2. there does not exist an outcome $z \in f(S)$ so that $z \leq s-(0.18040,0.18040,0.25512)$.

\section{Concluding Remarks on Decision Making with the Pareto Front Approximation}

We have introduced a way to construct a Pareto front approximation that can be used in decision making involving computationally expensive multiobjective optimization problems. As shown in (Hartikainen et al., 2011), the problem of choosing a preferred vector on the inherently nondominated Pareto front approximation $\mathscr{K}$ can be formulated as a multiobjective optimization problem

$$
\begin{array}{ll}
\min & \left(z_{1}, \ldots, z_{k}\right) \\
\text { s.t. } & z \in \operatorname{body}(\mathscr{K}) \\
\text { where } & z=\left(z_{1}, \ldots, z_{k}\right)^{T},
\end{array}
$$

which can be used as a surrogate to Problem (1). Additionally, it holds that

$$
\operatorname{PF}(\operatorname{body}(\mathscr{K}))=\operatorname{body}(\mathscr{K}),
$$

because $\mathscr{K}$ is inherently nondominated and, consequently, all vectors in the set $\operatorname{body}(\mathscr{K})$ are feasible solutions to problem (6). A preferred outcome of problem (6) is called a preferred vector on the approximation $\mathscr{K}$. Since problem (6) is a multiobjective optimization problem, any interactive multiobjective optimization methods can be employed to solve it.

The body of the inherently nondominated Pareto front approximation $\mathscr{K}$ can be parameterized and so problem (6) has a representation that can be input to e.g., the WWW-NIMBUS ${ }^{\circledR}$ implementation (Miettinen and Mäkelä, 2000, 2006) (available at http://nimbus.it.jyu.fi/) of the NIMBUS method (Miettinen and Mäkelä, 1995, 2006). Obviously, the parameters that are used for parameterizing the body of $\mathscr{K}$ are not meaningful to the decision maker but the vectors $z \in \operatorname{body}(\mathscr{K})$ are. The only drawback of this approach is that the parameters used for describing the different polytopes in the complex $\mathscr{K}$ are discrete and thus problem (6) has discrete 
variables in its decision space. In effect, the solvers used for solving the scalarizations employed by an interactive multiobjective optimization method must be able to handle discrete variables. Fortunately, e.g., WWW-NIMBUS ${ }^{\circledR}$ has global solvers that are able to do this.

The a priori error estimates are useful whenever a decision maker has chosen a preferred vector on the approximation. Whenever this happens, the decision maker can be given information about the actual Pareto optimal solutions in the form of error estimates. Having examined the error estimates, the decision maker can either choose to have the chosen point on the approximation projected on the actual Pareto front by means of the achievement scalarizing function (Wierzbicki, 1986) or not.

Future research on this topic is needed to implement the approximation approach given in this paper. For example, the computational expense of directly employing rules (R1) and (R2) grows exponentially when the number of objectives rises and smart computation schemes are needed to handle this. Second, we need to find an efficient way to solving problems (2) and (3). As further research, it is of interest to study which interactive multiobjective optimization methods and implementations are most suitable for solving problem (6) in real life problems and with real decision makers. The choice of the interactive method may obviously depend on the initial multiobjective optimization problem and also on the decision maker's desires. Finally, to make our approximation approach more versatile, we intend to examine how the continuity assumption in Section 2 can be relaxed.

Another direction of research is to study other uses of our approximation in multiobjective optimization. For example, in bilevel multiobjective optimization it is sometimes required to approximate the Pareto front of the lower level problem that is then brought to the upper level. Because of the inherent nondominance property, our approximation has potential to be efficient in this kind of use.

\section{References}

Bezerkin, V. E., Kamenev, G. K., and Lotov, A. V. (2006). Hybrid Adaptive Methods for Approximating a Nonconvex Multidimensional Pareto Frontier. Computational Mathematics and Mathematical Physics, 46, 1918-1931.

Boissonnant, J.-D. (1984). Geometric Structures for Three-Dimensional Shape Representation. ACM Transactions on Graphics, 3, 266-286.

Caratheodory, C. (1913). Bedingt Konvergente Reihen und Konvexe Systeme. Journal für die reine und angewandte Mathematik, 143, 128-175.

Coello Coello, C. A., Van Veldhuizen, D. A., and Lamont, G. B. (2007). Evolutionary Algorithms for Solving Multi-Objective Problems. Springer, New York.

Deb, K. (2001). Multi-Objective Optimization Using Evolutionary Algorithms. John Wiley \& Sons, Chichester.

Deb, K., Thiele, L., Laumanns, M., and Zitzler, E. (2002). Scalable Multi-Objective Optimization Test Problems. IEEE International Conference on E-Commerce Technology, 1, 825-830.

Edelsbrunner, H. (1987). Algorithms in Combinatorial Geometry. Springer, New York. 
Edelsbrunner, H. (1998). Shape Reconstruction with Delaunay Complex. In C. L. Lucchesi and A. V. Moura, editors, LATIN'98: Theoretical Informatics, pages 119132. Springer-Verlag Berlin, Heidelberg.

Edelsbrunner, H. and Shah, N. R. (1994). Triangulating Topological Spaces. In SCG '94: Proceedings of the Tenth Annual Symposium on Computational Geometry, pages 285-292. ACM, New York.

Efremov, R. V. and Kamenev, G. K. (2009). Properties of a Method for Polyhedral Approximation of the Feasible Criterion Set in Convex Multiobjective Problems. Annals of Operations Research, 166, 271-279.

Eskelinen, P., Miettinen, K., Klamroth, K., and Hakanen, J. (2010). Pareto Navigator for Interactive Nonlinear Multiobjective Optimization. OR Spectrum, 32, 211-227.

Fortune, S. (1997). Voronoi Diagrams and Delaunay Triangulations. In J. E. Goodman and J. O'Rourke, editors, Handbook of Discrete and Computational Geometry. CRC Press, Boca Raton.

Fukuda, K. (2004). Polyhedral Computation FAQ. Swiss Federal Institute of Technology, url = http://www.ifor.math.ethz.ch/ fukuda/polyfaq/polyfaq. html.

George, P.-L. and Borouchaki, H. (1998). Delaunay Triangulation and Meshing: Application to Finite Elements. Hermes, Paris.

Goel, T., Vaidyanathan, R., Haftka, R. T., Shyy, W., Queipo, N. V., and Tucker, K. (2007). Response Surface Approximation of Pareto Optimal Front in MultiObjective Optimization. Computer Methods in Applied Mechanics and Engineering, 196, 879-893.

Goodman, J. E. and O'Rourke, J., editors (1997). Discrete and Computational Geometry. CRC Press, Boca Raton.

Grünbaum, B. (1967). Convex Polytopes. Interscience Publishers, London.

Hartikainen, M., Miettinen, K., and Wiecek, M. M. (2011). Pareto Front Approximations for Decision Making with Inherent Non-dominance. In Y. Shi, S. Wang, G. Kou, and J. Wallenius, editors, New State of MCDM in the 21st Century, Selected Papers of the 20th International Conference on Multiple Criteria Decision Making 2009, pages 35-46. Springer-Verlag Berlin, Heidelberg.

Hasenjäger, M. and Sendhoff, B. (2005). Crawling Along the Pareto Front: Tales From the Practice. In The 2005 IEEE Congress on Evolutionary Computation (IEEE CEC 2005), pages 174-181, Piscataway, NJ. IEEE Press.

Keeney, R. L. and Raiffa, H. (1993). Decisions with Multiple Objectives. Cambridge University Press, Cambridge.

Laukkanen, T., Tveit, T.-M., Ojalehto, V., Miettinen, K., and Fogelholm, C.-J. (2010). An Interactive Multi-Objective Approach to Heat Exchanger Network Synthesis. Computers \& Chemical Engineering, 34, 943-952.

Lotov, A. V., Bushenkov, V. A., and Kamenev, G. A. (2004). Interactive Decision Maps. Kluwer Academic Publishers, Boston.

Luque, M., Ruiz, F., and Miettinen, K. (2011). Global Formulation for Interactive Multiobjective Optimization. OR Spectrum, 33, 27-48.

Martin, J., Bielza, C., and Insua, D. R. (2005). Approximating Nondominated Sets in Continuous Multiobjective Optimization Problems. Naval Research Logistics, 52 , 469-480. 
McMullen, P. (1970). The Maximum Number of Faces of a Convex Polytope. Mathematika, 17, 179-184.

Miettinen, K. (1999). Nonlinear Multiobjective Optimization. Kluwer Academic Publishers, Boston

Miettinen, K. and Mäkelä, M. (1995). Interactive Bundle-based Method for Nondifferentiable Multiobjective Optimization: NIMBUS. Optimization, 34, 231-246.

Miettinen, K. and Mäkelä, M. (2000). Interactive Multiobjective Optimization System WWW-NIMBUS on the Internet. Computers \& Operations Research, 27, 709-723.

Miettinen, K. and Mäkelä, M. M. (2006). Synchronous Approach in Interactive Multiobjective Optimization. European Journal of Operational Research, 170, 909922.

Miettinen, K., Ruiz, F., and Wierzbicki, A. P. (2008). Introduction to Multiobjective Optimization: Interactive Approaches. In J. Branke, K. Deb, K. Miettinen, and R. Slowinski, editors, Multiobjective Optimization: Interactive and Evolutionary Approaches, pages 27-57. Springer-Verlag Berlin, Heidelberg.

Monz, M. (2006). Pareto Navigation - Algorithmic Foundation of Interactive Multicriteria IMRT Planning. Ph.D. thesis, University of Kaiserslautern.

Rajan, V. T. (1994). Optimality of the Delaunay Triangulation in $\mathbb{R}^{d}$. Discrete and Computational Geometry, 12, 189- 202.

Ruzika, S. and Wiecek, M. M. (2005). Approximation Methods in Multiobjective Programming. Journal of Optimization Theory and Applications, 126, 473-501.

Sawaragi, Y., Nakayama, H., and Tanino, T. (1985). Theory of Multiobjective Optimization. Academic Press, Orlando.

Schandl, B., Klamroth, K., and Wiecek, M. M. (2001). Norm-Based Approximation in Bicriteria Programming. Computational Optimization and Applications, 20, 23 -42 .

Schandl, B., Klamroth, K., and Wiecek, M. M. (2002). Norm-Based Approximation in Multicriteria Programming. Computers \& Mathematics with Applications, 44, $925-942$.

Shenton, D. and Cendes, Z. (1985). Three-Dimensional Finite Element Mesh Generation Using Delaunay Tesselation. IEEE Transactions on Magnetics, 21, 2535 2538.

Steuer, R. E. (1986). Multiple Criteria Optimization: Theory, Computation and Application. John Wiley \& Sons, New York.

Steuer, R. E. (1989). The Tchebycheff Procedure of Interactive Multiple Objective Programming. In B. Karpak and S. Zionts, editors, Multiple Criteria Decision Making and Risk Analysis Using Micro Computers. Springer-Verlag Berlin, Heidelberg.

Steuer, R. E. and Choo, E.-U. (1983). An Interactive Weighted Tchebycheff Procedure for Multiple Objective Programming. Mathematical Programming, 26, 326344.

Vegter, G. (1997). Computational Topology. In J. E. Goodman and J. O'Rourke, editors, Handbook of Discrete and Computational Geometry, pages 719-742. CRC Press, Boca Raton. 
Wierzbicki, A. P. (1986). On the Completeness and Constructiveness of Parametric Characterizations to Vector Optimization Problems. OR Spectrum, 8, 73-87.

Yu, P. L. and Zeleny, M. (1975). The Set of All Nondominated Solutions in Linear Cases and a Multicriteria Simplex Method. Journal of Mathematical Analysis and Applications, 49, 430-468. 